\title{
Caspase cleavage of vimentin disrupts intermediate filaments and promotes apoptosis
}

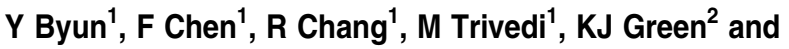 \\ VL Cryns*,1 \\ ${ }^{1}$ Center for Endocrinology, Metabolism and Molecular Medicine, Department of \\ Medicine, Northwestern University Medical School, Chicago, IL 60611, USA \\ 2 Department of Pathology, Northwestern University Medical School, Chicago, \\ IL 60611, USA \\ * Corresponding author: Vincent Cryns, Division of Endocrinology, Tarry 15- \\ 755, Northwestern University Medical School, 303 E. Chicago Ave., Chicago \\ IL 60611, USA. Tel: (312) 503-0644; Fax: (312) 908-9032 \\ E-mail:v-cryns@northwestern.edu.
}

Received 17.7.00; revised 30.11.00; accepted 19.12.00

Edited by $Y$ Lazebnik

\begin{abstract}
Caspases are key mediators of apoptosis. Using a novel expression cloning strategy we recently developed to identify cDNAs encoding caspase substrates, we isolated the intermediate filament protein vimentin as a caspase substrate. Vimentin is preferentially cleaved by multiple caspases at distinct sites in vitro, including Asp ${ }^{85}$ by caspases-3 and -7 and Asp ${ }^{259}$ by caspase-6, to yield multiple proteolytic fragments. Vimentin is rapidly proteolyzed by multiple caspases into similar sized fragments during apoptosis induced by many stimuli. Caspase cleavage of vimentin disrupts its cytoplasmic network of intermediate filaments and coincides temporally with nuclear fragmentation. Moreover, caspase proteolysis of vimentin at $\mathrm{Asp}^{\mathbf{8 5}}$ generates a pro-apoptotic amino-terminal fragment whose ability to induce apoptosis is dependent on caspases. Taken together, our findings suggest that caspase proteolysis of vimentin promotes apoptosis by dismantling intermediate filaments and by amplifying the cell death signal via a pro-apoptotic cleavage product. Cell Death and Differentiation (2001) 8, 443-450.
\end{abstract}

Keywords: caspases; proteases; cytoskeleton; intermediate filaments; vimentin

Abbreviations: zVAD-fmk, z-Val-Ala-Asp(OMe)-fmk; TNF, tumor necrosis factor; PARP, poly(ADP-ribose) polymerase; kDa, kilodaltons; SDS-PAGE, sodium dodecyl sulfate-polyacrylamide gel electrophoresis; mAb, monoclonal antibody; PCR, polymerase chain reaction; GFP, green fluorescent protein

\section{Introduction}

Apoptosis or programmed cell death is a genetically regulated cellular suicide response that results in a series of distinctive morphological events including membrane blebbing, cellular rounding, nuclear condensation/fragmentation and subsequent encapsulation of these fragments into 'apoptotic bodies' that are degraded by neighboring cells. ${ }^{1}$ Caspases are a conserved family of cysteine proteases with aspartate substrate specificity that play a crucial role in the execution of apoptosis by cleaving and modifying the activity/function of diverse intracellular proteins. ${ }^{2,3}$ Indeed, caspase proteolysis of several proteins, including Gas2, gelsolin, fodrin, the nuclear lamins, keratins 18 and 19 , and $\beta$-catenin, has been implicated in the dramatic cytoskeletal reorganization that occurs during apoptotic cell death. ${ }^{4-14}$ For instance, caspase cleavage of Gas2 and gelsolin disrupts actin filaments and directly promotes many of the morphological manifestations of apoptosis. $^{4,5}$ In addition, several structurally related intermediate filament proteins, including the lamins ( $A, B$ and $C)$ and cytokeratins 18 and 19, are cleaved by caspase- 6 at a conserved aspartate residue in their respective L1-2 linker domains. $^{8-13}$ Caspase cleavage of the lamins is functionally linked to the disassembly of the nucleus during apoptosis. ${ }^{10}$ Clearly, the identity of additional cytoskeletal caspase targets and the functional significance of their proteolysis has yet to be elucidated.

In an effort to delineate the molecular mechanisms by which caspases induce apoptosis, we have recently developed a novel expression cloning strategy to systematically identify cDNAs encoding caspase substrates. ${ }^{15,16}$ Using this approach, we report here the isolation of the intermediate filament protein vimentin as a downstream caspase target. We demonstrate that vimentin is cleaved by multiple caspases at distinct sites, including $A_{s p}{ }^{85}$ by caspases-3 and -7 and $A \mathrm{sp}^{259}$ by caspase- 6 , both in vitro and during apoptosis. We also show that caspase proteolysis of vimentin has two important functional consequences: (i) the irreversible disruption of intermediate filaments; and (ii) the generation of a pro-apoptotic amino-terminal cleavage product (amino acids $1-85$ ) that serves to amplify the cell death signal.

\section{Results}

Isolation of vimentin as a caspase-3 substrate by small pool expression cloning

We have recently used a novel in vitro expression cloning strategy to systematically identify cDNAs encoding putative caspase substrates that are proteolyzed during apoptotic cell death. ${ }^{15,16}$ In the current screen, we incubated ${ }^{35}$ S-labeled protein pools derived from small pools of a human heart cDNA library (48 cDNAs/pool) with recombinant caspases-3 and -8. As shown in Figure $1 \mathrm{~A},{ }^{35} \mathrm{~S}$-labeled protein pool 34 contained a $57 \mathrm{kDa}$ protein (indicated by asterisk) that was specifically cleaved by caspase-3 (C3) into an approximately 48 kDa 
fragment (indicated by arrow); this proteolytic fragment was not observed when protein pool 34 was incubated with control buffer (C) or caspase-8 (C8). The proteolytic activity of caspase-3 (C3) and caspase-8 (C8) was verified by incubating these proteases with ${ }^{35} \mathrm{~S}$-labeled poly(ADPribose) polymerase (PARP), a well characterized caspase substrate: ${ }^{18}$ caspase-3 (C3) and, to a lesser extent, caspase8 (C8) cleaved PARP into its signature $85 \mathrm{kDa}$ fragment into smaller pools and the corresponding ${ }^{35} \mathrm{~S}$-labeled protein pools were re-examined as above. As shown in Figure 1C, a single cDNA encoding a $57 \mathrm{kDa}$ protein that was cleaved by caspase-3 (C3) into an approximately $48 \mathrm{kDa}$ fragment was isolated. Sequence analysis of this cDNA revealed that it was human vimentin, an abundant type III intermediate filament protein that is expressed in mesenchymal tissues and in a broad spectrum of carcinomas. ${ }^{19,20}$

\section{Vimentin is proteolyzed at several sites including Asp ${ }^{85}$ and $A^{2}{ }^{259}$ by multiple caspases in vitro}

Having isolated vimentin by virtue of its sensitivity to proteolysis by caspase-3 in vitro, we next wanted to determine whether vimentin was cleaved by other caspases (Figure 1B). cDNA pool 34 was then progressively subdivided

in vitro. As demonstrated in Figure $2 \mathrm{~A}$, vimentin was most efficiently proteolyzed by caspases-3, -6 and -7 into distinct patterns of proteolytic products in vitro (indicated by arrows and designated I-V); the proteolytic activity of each caspase was verified by incubation with a known ${ }^{35} \mathrm{~S}$-labeled substrate (data not shown). Caspase-3 (C3) preferentially cleaved vimentin to generate fragments II (the above-noted $48 \mathrm{kDa}$ product) and III (fragments I, IV and V were minor products); fragment II was also the most abundant product resulting from vimentin cleavage by caspase-7 (C7). In contrast, caspase- 6 (C6) cleaved vimentin most efficiently into fragments I, IV and $\mathrm{V}$ (fragment II was less efficiently produced by caspase- 6 than by caspases-3 or -7). To determine vimentin's major caspase cleavage sites in vitro, we examined its sequence and identified a potential caspase- 3 cleavage site (DXXD motif) that would generate fragment II (DSVD ${ }^{85}-F$ in the non-helical amino-terminus) and a potential caspase- 6 site (V/IXXD motif) that would produce fragments IV and V (IDVD ${ }^{259}-\mathrm{V}$ in the L1-2 domain). ${ }^{21,22}$ We next selectively altered these aspartic acid residues by site-directed mutagenesis to create two mutant vimentin constructs: D85E and D259E. As shown in Figure $2 \mathrm{~B}$, incubation of ${ }^{35} \mathrm{~S}$-labeled D85E with caspases-3 and -7 failed to generate proteolytic fragment II that was observed when ${ }^{35} \mathrm{~S}$-labeled wild-type (WT) or D259E mutant were incubated with these caspases. Moreover, ${ }^{35} \mathrm{~S}$-labeled

$\mathbf{A}$

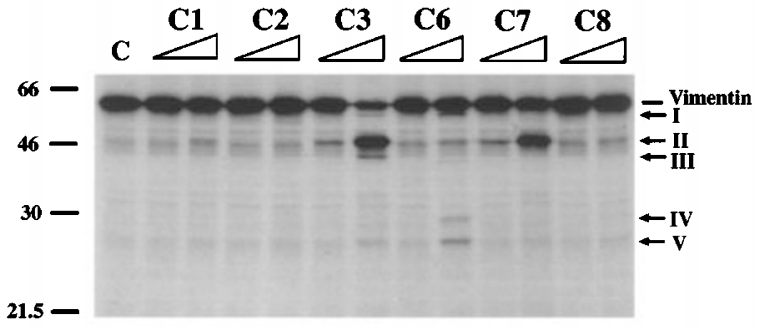

B

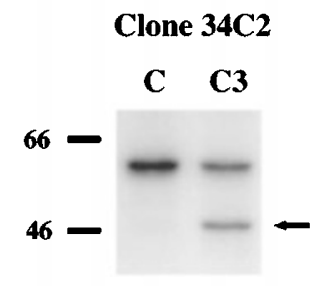

\section{$21.5-$}

Figure 1 Isolation of vimentin as a caspase-3 substrate in vitro by small pool expression cloning. (A) ${ }^{35} \mathrm{~S}$-labeled protein pool 34 contains a $57 \mathrm{kDa}$ protein (indicated by asterisk) that is specifically cleaved into an approximately $48 \mathrm{kDa}$ fragment (indicated by arrow) by caspase-3 (C3), but not by buffer control (C) or caspase-8 (C8). (B) Specific proteolysis of ${ }^{35}$ S-labeled PARP into its characteristic $85 \mathrm{kDa}$ cleavage fragment (indicated by arrow) by caspase- 3 , and to a lesser extent, by caspase-8. (C) Isolation of a single cDNA from poo 34 that encodes a $57 \mathrm{kDa}$ protein that is cleaved by caspase-3 into the previously noted $48 \mathrm{kDa}$ fragment. Sequence analysis of this cDNA revealed that it is human vimentin. Small pool expression cloning was performed as described previously ${ }^{15,16}$ and in Materials and Methods. The molecular mass of markers is indicated at the left of each panel 
D259E was resistant to proteolysis by caspase- 6 at this site: fragments IV and V produced by caspase- 6 cleavage of wildtype or D85E vimentin were not observed upon incubation of D259E with this protease. Taken together, these studies indicate that vimentin is preferentially cleaved by caspases-3 and -7 at $\mathrm{Asp}^{85}$ and by caspase- 6 at $\mathrm{Asp}^{259}$ in vitro; the identity of vimentin's additional caspase cleavage site(s) has yet to be determined. Interestingly, vimentin's caspase- 6 cleavage site $\left(\mathrm{Asp}^{259}\right)$ in its L1-2 linker domain corresponds precisely to the caspase cleavage sites previously reported in the structurally related intermediate filament proteins, the lamins and cytokeratins 18 and $19 .^{8-13}$

\section{Vimentin is specifically cleaved by caspases during the induction of apoptosis in vivo}

To determine whether vimentin is proteolyzed by caspases during apoptosis in vivo, we treated HeLa cells with TNF- $\alpha$ / cycloheximide or staurosporine for variable time periods and examined cell lysates for evidence of vimentin cleavage by immunoblotting. As shown in Figure 3A, vimentin was rapidly proteolyzed into an approximately $48 \mathrm{kDa}$ fragment (indicated by arrow and designated II) within $2 \mathrm{~h}$ of treating cells with either TNF- $\alpha /$ cycloheximide or staurosporine. This cleavage occurred early during the induction of apoptosis when fewer than $10 \%$ of treated cells had condensed or fragmented nuclei indicative of apoptotic cell death (the percentage of cells with apoptotic nuclei at each time point is indicated at the bottom of Figure $3 \mathrm{~A}$ ). Additional vimentin fragments (indicated by arrows and labeled I, III-V) were subsequently generated during the induction of apoptosis by these stimuli; fragments I, IV and $\mathrm{V}$ were observed only after prolonged exposure of immunoblots from TNF- $\alpha$ treated cells (data not shown). Vimentin was also cleaved into similar sized fragments in Jurkat cells induced to undergo apoptosis by treatment with etoposide or anti-Fas mAb (data not shown). Importantly, the vimentin cleavage products observed during the induction of apoptosis in vivo were of the identical (fragments I-III) or similar (fragments IV and V) size as the corresponding fragments generated by caspase cleavage of vimentin in vitro (data not shown), thereby strongly suggesting that caspases are responsible for its apoptotic cleavage. Further support for this notion comes from the observation that vimentin's cleavage during apoptosis coincided closely with that of the well characterized caspase cytoskeletal target $\alpha$-fodrin ${ }^{6,7}$ and with pro-caspase-3 proteolytic activation (manifested as a reduction in proenzyme intensity and/or processing into its p20 and p17 subunits) (Figure 3A). Moreover, as demonstrated in Figure $3 \mathrm{~B}$, the broad-spectrum caspase-inhibitor zVAD-fmk antagonized staurosporine-induced vimentin proteolysis and apoptotic cell death (as indicated by diminished nuclear fragmentation). Of note, the various vimentin cleavage products differed in their sensitivity to inhibition by zVAD-fmk: fragment III was completely inhibited by $10 \mu \mathrm{M}$, fragment IV by $50 \mu \mathrm{M}$, while fragments I and II were almost completely inhibited by $250 \mu \mathrm{M}$. Fragment $\mathrm{V}$ was faintly observed after prolonged exposure of immunoblots only in staurosporine-treated cells that had not received any zVADfmk (data not shown). Taken together, these results indicate unequivocally that vimentin is proteolytically cleaved by
A

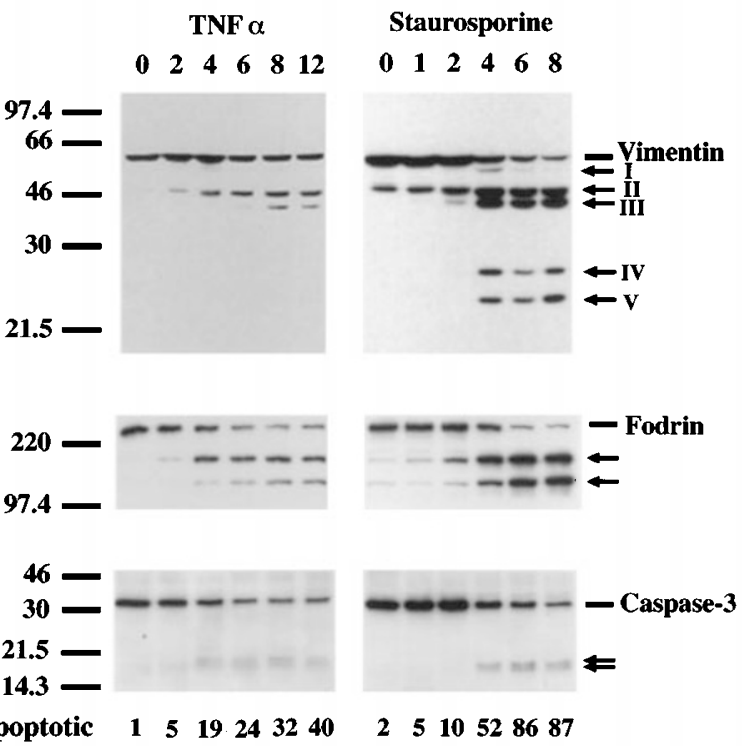

B

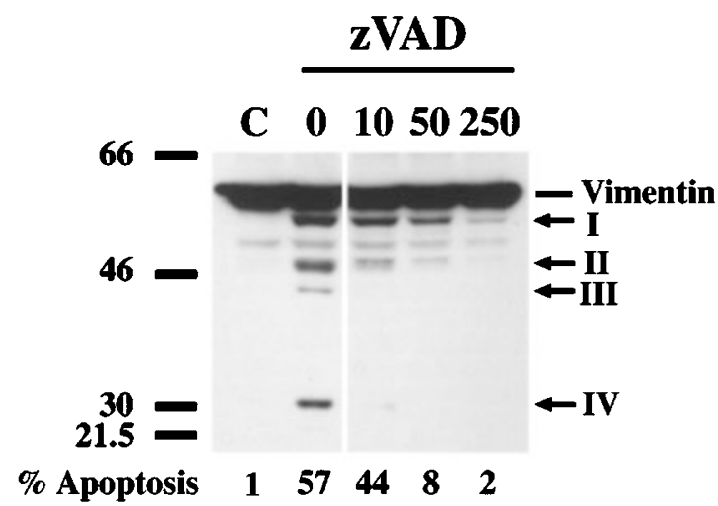

Figure 3 Vimentin is specifically cleaved by caspases during the induction of apoptosis in vivo. (A) Vimentin is rapidly proteolyzed during TNF- $\alpha$ and staurosporine-induced apoptosis into multiple fragments of similar size to those generated in vitro by caspase cleavage; the time course of vimentin's apoptotic cleavage is similar to that of $\alpha$-fodrin and pro-caspase-3. HeLa cells were treated with $10 \mathrm{ng} / \mathrm{ml} \mathrm{TNF}-\alpha$ and $1 \mu \mathrm{g} / \mathrm{ml}$ cycloheximide for $0-12 \mathrm{~h}$ or with $1 \mu \mathrm{M}$ staurosporine for $0-8 \mathrm{~h}$. (B) $z V A D$-fmk inhibits staurosporine-induced vimentin proteolysis. HeLa cells were preincubated with ZVAD-fmk $(0-$ $250 \mu \mathrm{M})$ for $2 \mathrm{~h}$, and then treated for an additional $4 \mathrm{~h}$ with $1 \mu \mathrm{M}$ staurosporine. Cell lysates were analyzed by immunoblotting and the percentage of apoptotic cells (indicated at the bottom) was determined by nuclear morphology as detailed in Materials and Methods. The molecular mass in $\mathrm{kDa}$ is indicated at the left of each panel

multiple caspases (with different sensitivities to inhibition by zVAD-fmk) acting at distinct time points during the execution of apoptotic cell death.

\section{Caspase cleavage of vimentin disrupts intermediate filaments during apoptosis}

To assess the functional consequences of vimentin's proteolysis by caspases, we examined the cellular distribu- 
tion of vimentin by indirect immunofluorescence during the induction of apoptosis. As shown in Figure 4, vimentin in untreated, control HeLa cells (left-hand, upper panel) was organized into an extensive network of cytoplasmic intermediate filaments. However, after $3 \mathrm{~h}$ of treatment with TNF- $\alpha$ and cycloheximide, vimentin intermediate filaments were dismantled into punctate or granular aggregates in many cells (indicated by arrows, middle upper panel). This striking cytoskeletal event coincided temporally with nuclear fragmentation (indicated by arrows, middle lower panel): under these conditions, all cells with disrupted vimentin filaments were clearly apoptotic by nuclear morphology. The caspase inhibitor zVAD-fmk antagonized vimentin intermediate filament disruption and nuclear fragmentation induced by TNF- $\alpha$ (right-hand, upper and lower panels, respectively), thereby confirming the critical role that caspases play in vimentin filament disassembly.

\section{Caspase proteolysis of vimentin at $\mathrm{Asp}^{85}$ generates a pro-apoptotic amino-terminal product that amplifies the cell death signal}

Because caspases initially cleave vimentin at $A s{ }^{85}$ during the induction of apoptosis, we postulated that the aminoterminal cleavage product corresponding to amino acids 185 ('truncated' vimentin) might contribute to the execution of apoptosis. As shown in Figure 5A, GFP-tagged vimentin assembles into extensive intermediate filaments surrounding normal nuclei, while GFP-tagged truncated vimentin is assembly incompetent and induces cellular rounding, cytosolic shrinkage and nuclear condensation/fragmentation. Indeed, GFP-tagged truncated vimentin often forms granular aggregates in cells that resemble those seen in TNF$\alpha$-treated cells (see Figure 5B, left-hand, upper panel); cells bearing such aggregates commonly have apoptotic nuclear

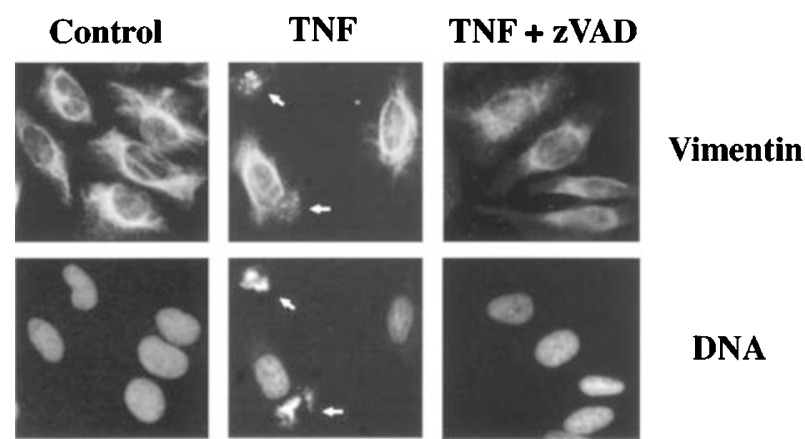

Figure 4 Caspase cleavage of vimentin disrupts intermediate filaments during apoptosis. TNF- $\alpha$ induces the rearrangement of vimentin intermediate filaments into punctate, granular aggregates (indicated by arrows); this cytoskeletal event coincides temporally with the induction of nuclear fragmentation (indicated by arrows). The caspase inhibitor zVAD-fmk blocks both vimentin filament disruption and nuclear fragmentation. HeLa cells were untreated (control, left-hand panels) or preincubated with DMSO (middle panels) or $250 \mu \mathrm{M}$ zVAD-fmk (right-hand panels) for $1 \mathrm{~h}$, and then treated for an additional $3 \mathrm{~h}$ (DMSO) or $5 \mathrm{~h}$ (ZVAD-fmk) with $10 \mathrm{ng} / \mathrm{ml} \mathrm{TNF}-\alpha$ and $1 \mu \mathrm{g} / \mathrm{ml}$ cycloheximide. Vimentin intermediate filaments (upper panels) and nuclear morphology (lower panels) were visualized as detailed in Materials and Methods morphology (Figure 5B, left-hand, lower panel). Moreover, truncated vimentin interferes with the assembly of wild-type vimentin into intermediate filaments in co-transfection experiments (Figure 5B, right-hand, upper panel). As demonstrated in Figure $5 \mathrm{C}$, transient expression of GFPtruncated vimentin but not full-length vimentin induces apoptosis in vimentin-positive HeLa cells and in vimentinnegative MCF-7 cells (which also lack caspase-3). Interestingly, apoptosis induced by truncated vimentin requires caspases: the broad spectrum caspase inhibitor zVAD-fmk antagonizes truncated vimentin-induced cell death. To delineate which caspases might play a role in apoptosis induced by truncated vimentin, we co-transfected HeLa cells with truncated vimentin and a variety of anti-apoptotic cDNAs. As shown in Figure 5D, dominant negative caspase-8 and dominant negative FADD, specific inhibitors of caspase- $8,{ }^{23}$ CrmA (an inhibitor of caspases-1 and $-8^{24}$ ), p35 (a nonselective caspase inhibitor) and $\mathrm{Bcl}-\mathrm{x}_{\mathrm{L}}$ antagonize cell death induced by truncated vimentin. Hence, truncated vimentin induces apoptosis in cells by a caspase-dependent mechanism, and caspase- 8 may be particularly important in mediating its pro-apoptotic actions.

\section{Discussion}

We have demonstrated that vimentin, an abundant type III intermediate filament protein, is rapidly proteolyzed by multiple caspases at several sites during apoptosis induced by diverse stimuli. Vimentin is initially cleaved at $\mathrm{Asp}^{85}$ $\left(D_{S V D}{ }^{85}-\mathrm{F}\right)$ by a caspase-3/-7-like protease and subsequently at additional sites, including $A_{s p}{ }^{259}$ (IDVD ${ }^{259}-\mathrm{V}$ ) which is cleaved by a caspase-6-like protease. Importantly, vimentin is proteolyzed at $\mathrm{Asp}^{85}$ during the induction of apoptosis when the vast majority $(>90 \%)$ of cells are still viable; cleavage at this site coincides temporally with caspase-3 proteolytic activation and cleavage of the caspase cytoskeletal target $\alpha$-fodrin. ${ }^{6,7}$ Consequently, vimentin proteolysis precedes the dramatic reorganization of the cytoskeleton that typifies apoptotic cell death and could therefore play a role in its execution.

Although other studies have indicated that vimentin is cleaved in response to certain apoptotic stimuli, ${ }^{25,26}$ the present study provides a more detailed characterization of the molecular mechanisms underlying its apoptotic cleavage. Moreover, our results differ from those of Morishima who reported that vimentin is first cleaved at $A s p^{259}$ by a caspase-8-like protease and subsequently at $\mathrm{Asp}^{85}$ by caspase-3-like protease. ${ }^{27}$ Although the explanation for this discrepancy is unclear, we have demonstrated unambiguously that $\mathrm{Asp}^{259}$ in vimentin is readily cleaved by caspase6 in vitro but not by limited amounts ( 2.5 or $25 \mathrm{ng}$ ) of caspase-8. In contrast, Morishima shows only that caspase-8 (amount unspecified) cleaves $\mathrm{Asp}^{259}$ in vitro. Because isoleucine in the $\mathrm{P} 4$ substrate recognition sequence at $\mathrm{Asp}^{259}\left(\mathrm{IDVD}^{259}-\mathrm{V}\right)$ is likely to be well tolerated by either caspase- 6 or $-8,{ }^{21,22}$ we postulate that although caspase-8 may be able to cleave $\mathrm{Asp}^{259}$ in vitro (particularly when caspase- 8 is present at high concentrations), caspase- 6 is the primary caspase responsible for its proteolysis at this site. 
A

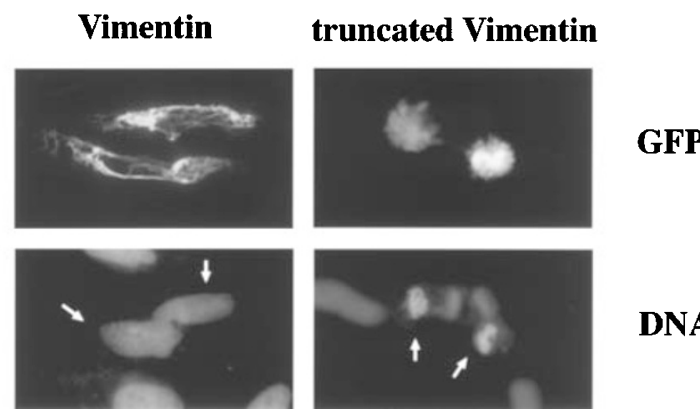

C

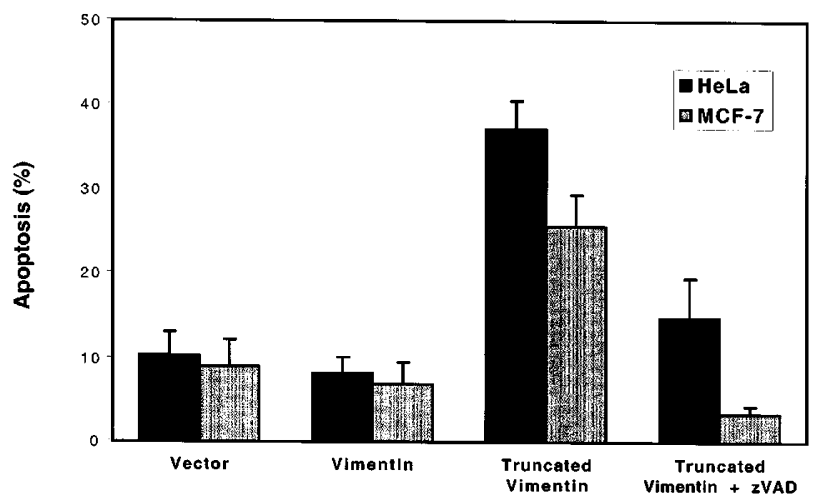

B
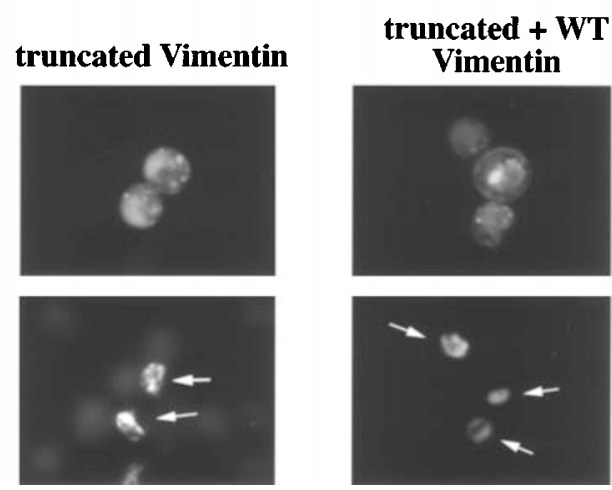

GFP

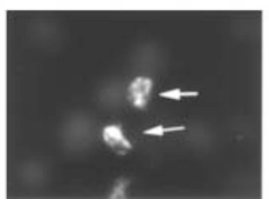

DNA

D

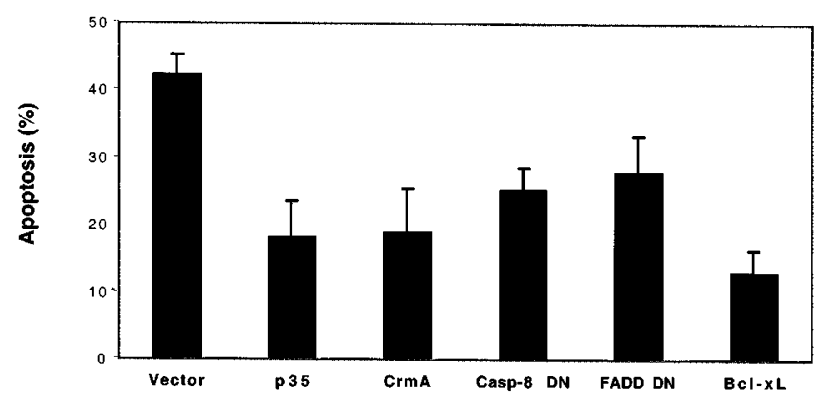

Figure 5 Truncated vimentin interferes with intermediate filament assembly and induces apoptosis by a caspase-dependent mechanism. (A) HeLa cells transiently transfected with GFP-vimentin assemble into intermediate filaments surrounding normal nuclei (left-hand, upper and lower panels), while GFP-truncated vimentin is assembly incompetent and induces cellular rounding, cytosolic shrinkage and nuclear condensation/fragmentation (right-hand, upper and lower panels). (B) Vimentin-negative MCF-7 cells were transiently transfected with GFP-truncated vimentin alone or co-transfected with GFP-truncated vimentin and GFP-wild-type vimentin ( $1 \mu \mathrm{g}$ each). Truncated vimentin often forms granular aggregates in MCF-7 cells (left-hand, upper panel) and interferes with wild-type vimentin's assembly into intermediate filaments (right-hand, upper panel); MCF-7 cells containing granular aggregates often have apoptotic nuclei (lower panels). (C) HeLa and MCF-7 cells were transfected with $1 \mu \mathrm{g}$ of pEGFP-N1 plasmid containing empty vector, full-length or truncated vimentin (the latter in the presence or absence of $100 \mu \mathrm{M}$ zVAD-fmk). Twenty-four hours later, GFP-positive cells were scored for apoptosis by nuclear morphology as detailed in 'Materials and Methods'. (D) HeLa cells were co-transfected with $0.4 \mu \mathrm{g}$ of pEGFP-N1 plasmid containing truncated vimentin and $1.2 \mu \mathrm{g}$ of various anti-apoptotic cDNAs (p35, CrmA, caspase-8 dominant negative (Casp-8 DN), FADD dominant negative (FADD DN), or Bcl- $\mathrm{x}_{\mathrm{L}}$ ). GFP-positive cells were scored for apoptosis as above. The data in $(\mathbf{C})$ and $(\mathbf{D})$ represent the mean of at least triplicate experiments \pm S.D.

Vimentin, then, can be added to the list of intermediate filament proteins, including the nuclear lamins and cytokeratins 18 and 19, which are cleaved by caspases during apoptotic cell death. ${ }^{8-13}$ Like these other intermediate filament proteins, vimentin is cleaved by caspase- 6 or a caspase-6-like protease at a conserved motif in its L1-2 domain (IDVD in vimentin and VEV/ID in the lamins and cytokeratins). Caspase- 6 cleavage of the nuclear lamins at the corresponding site directly contributes to the apoptotic destruction of the nucleus: selective inhibition of lamin proteolysis by introducing a cleavage-resistant mutant lamin into cells delays chromatin condensation and DNA fragmentation. ${ }^{10}$ However, introduction of the corresponding vimentin D259E mutant construct into MCF-7 cells (which lack vimentin) did not confer protection against apoptotic cell death ( $\mathrm{M}$ Trivedi and V Cryns, unpublished data), likely reflecting the sensitivity of this mutant vimentin protein to caspase cleavage at additional sites. Indeed, vimentin's apoptotic cleavage at multiple sites outside the L1-L2 linker domain distinguishes it from the nuclear lamins.
We have also demonstrated that caspase cleavage of vimentin has important functional consequences, including the dismantling of vimentin intermediate filaments into granular aggregates that bear a striking resemblance to those generated by caspase proteolysis of cytokeratins. $^{12,28}$ Although phosphorylation of vimentin at Ser ${ }^{55}$ leads to filament disassembly during mitosis, ${ }^{29}$ our finding that the apoptotic dismantling of vimentin filaments is zVAD-fmk-inhibitable indicates that caspases play an essential role in this process, independent of any potential phosphorylation events. Given the evidence that several intermediate filament proteins (some with different patterns of tissue expression) are caspase substrates, caspase dismantling of the intermediate filament network is a central theme in the execution of apoptosis. It seems likely that the proteolytic disassembly of this rigid cytosolic network may facilitate many of the morphological manifestations of apoptosis, including cellular rounding and packaging the debris of dying cells into apoptotic bodies. 
Importantly, we have demonstrated for the first time that caspase proteolysis of vimentin plays an active role in the execution of apoptosis. Specifically, the initial cleavage of vimentin at $A s p^{85}$ by a caspase-3-like protease during the induction of apoptosis generates an amino-terminal cleavage product (truncated vimentin) that is sufficient to induce apoptosis. Truncated vimentin is unable to assemble into intermediate filaments; instead, it often forms granular aggregates that closely resemble those seen in apoptotic cells and/or assumes a diffuse cytoplasmic pattern of staining. Truncated vimentin also disrupts the assembly of wild-type vimentin into intermediate filaments, a finding that is consistent with the observation that synthetic peptides derived from vimentin's amino-terminus interfere with intermediate filament assembly. ${ }^{30}$ Although we have yet to detect truncated vimentin directly in apoptotic cells, we postulate that this proteolytic fragment is likely to be shortlived, perhaps reflecting proteasomal degradation of this fragment that is prone to aggregation. Nevertheless, given the highly dynamic nature of vimentin filaments that are constantly undergoing subunit assembly and disassembly $\left(t_{1 / 2}\right.$ approximately $\left.5 \mathrm{~min}\right),{ }^{31}$ even a short-lived inhibitor of filament assembly would likely have profound cytoskeletal effects. Furthermore, we have also demonstrated that apoptosis induced by truncated vimentin is dependent on caspase activity, since non-specific caspase inhibitors (zVAD-fmk, p35 and CrmA) antagonize truncated vimentin-induced cell death. Caspase-8 may be a particularly important mediator of truncated vimentin-induced cell death: specific inhibitors of caspase-8 (dominant negative forms of caspase-8 or FADD) partially block apoptosis induced by truncated vimentin. These findings indicate that caspase cleavage of vimentin amplifies the cell death signal by generating an amino-terminal proteolytic product that, in turn, activates more caspases, thereby creating a positive feedback loop. A similar positive feedback loop has been described for other caspase substrates such as the kinase MEKK-1. ${ }^{32}$ Additional studies will be needed to clarify the mechanism(s) by which truncated vimentin activates caspases and whether the granular aggregates of vimentin participate in this process.

\section{Materials and Methods}

\section{Cell lines and reagents}

HeLa and MCF-7 cells were grown in DMEM (Life Technologies, Inc.) with $10 \%$ heat-inactivated fetal calf serum. $z$-Val-Ala-Asp(OMe)-fmk (zVAD-fmk) was purchased from Enzyme Systems Products and human recombinant tumor necrosis factor (TNF)- $\alpha$ from R\&D Systems. Bisbenzimide (Hoescht \#33258) and staurosporine were obtained from Sigma.

\section{Small pool expression cloning}

A human heart cDNA library in the pcDNA3 vector (Invitrogen) was subdivided into small pools (48 clones/pool); these pools were screened for cDNAs encoding putative caspase substrates as we have described previously except that recombinant caspase-3 $(50 \mathrm{ng})$ and $-8(100 \mathrm{ng})$ were used instead of apoptotic extracts. ${ }^{15,16}$

\section{Proteolytic cleavage of human vimentin in vitro}

${ }^{35} \mathrm{~S}$-labeled human vimentin was prepared by in vitro transcription/ translation of pcDNA3-vimentin using the TnT T7 Quick Coupled Transcription/Translation system (Promega) according to the manufacturer's instructions. ${ }^{35}$ S-labeled vimentin was then incubated with control buffer, 2.5 or $25 \mathrm{ng}$ of recombinant caspases-1, $-2,-3,-6$, -7 , or -8 for $1 \mathrm{~h}$ at $37^{\circ} \mathrm{C}$ and the cleavage reaction products analyzed as detailed previously. ${ }^{6,17}$ The catalytic activity of each caspase was confirmed by incubation with a known ${ }^{35} \mathrm{~S}$-labeled substrate.

\section{Determination of vimentin's caspase cleavage sites in vitro by site-directed mutagenesis}

Two mutant human vimentin constructs specifically altered at potential caspase cleavage sites, D85E and D259E, were made using the QuickChange Site-Directed Mutagenesis kit (Stratagene) according to the manufacturer's instructions with the following oligonucleotide primers: 5'-GGACTCGGTGGAATTCTCGCTGGCCG-3' and 5'-CGGCCAGCGAGAATTCCACCGAGTCC-3' (D85E) and 5'-CCAAATCGATGTGGAGGTTTCCAAGCCTGACC-3' and 5'-GGTCAGGCTTGGAAACCTCCACATCGATTTGG-3' (D259E). Mutations were verified by automated DNA sequencing of both strands. These mutant vimentin constructs were then ${ }^{35} \mathrm{~S}$-labeled, incubated with $25 \mathrm{ng}$ of caspases $-3,-6$ or -7 , and analyzed as above.

\section{Induction and quantitation of apoptosis}

HeLa cells were grown on glass coverslips and were treated with $10 \mathrm{ng} / \mathrm{ml} \mathrm{TNF}-\alpha$ and $1 \mu \mathrm{g} / \mathrm{ml}$ cycloheximide for $0,2,4,6,8$, or $12 \mathrm{~h}$ or with $1 \mu \mathrm{M}$ staurosporine for $0,1,2,4,6$, or $8 \mathrm{~h}$. For inhibitor studies, HeLa cells were preincubated with zVAD-fmk $(0,10,50$ or $250 \mu \mathrm{M})$ for $2 \mathrm{~h}$, and then treated for an additional $4 \mathrm{~h}$ with $1 \mu \mathrm{M}$ staurosporine. Following treatment, cells were fixed in $100 \%$ methanol for $2 \mathrm{~min}$ at $-20^{\circ} \mathrm{C}$ and nuclei were stained with $10 \mu \mathrm{g} / \mathrm{ml}$ Hoescht \#33258 for $30 \mathrm{~min}$. The percentage of apoptotic cells was determined by scoring for fragmented/condensed nuclei as visualized by fluorescence microscopy. For each treatment condition, at least 200 nuclei were scored; all experiments were performed in triplicate.

\section{Immunoblotting}

Whole cell lysates were prepared and examined by immunoblotting as described previously ${ }^{6}$ using the following antibodies: vimentin mAb V9 (Sigma, 1:1000 dilution), $\alpha$-fodrin mAb 1622 (Chemicon, 1:1000 dilution) and caspase-3 mAb (Transduction Laboratories, 1:1000 dilution).

\section{Indirect immunofluorescence}

HeLa cells were grown to approximately $50 \%$ confluence on glass coverslips: control cells were untreated, while other cells were preincubated with vehicle (DMSO) or $250 \mu \mathrm{M} z V A D-f m k$ for $1 \mathrm{~h}$ prior to treatment with $10 \mathrm{ng} / \mathrm{ml} \mathrm{TNF} \alpha$ and $1 \mu \mathrm{g} / \mathrm{ml}$ cycloheximide for an additional $3 \mathrm{~h}$ (vehicle-treated) or $5 \mathrm{~h}$ (zVAD-fmk-treated). Cells were subsequently fixed in $100 \%$ methanol for $2 \mathrm{~min}$ at $-20^{\circ} \mathrm{C}$, washed in PBS, and incubated with vimentin V9 mAb ( $1: 20$ dilution in PBS) for $2 \mathrm{~h}$ at $37^{\circ} \mathrm{C}$. After washing in PBS, cells were incubated with fluorescein-conjugated goat affinity purified $A b$ to mouse IgG (ICN Pharmaceuticals, 1:20 dilution in PBS) and $10 \mu \mathrm{g} / \mathrm{ml}$ Hoescht \#33258 for $30 \mathrm{~m}$ at $37^{\circ} \mathrm{C}$, and then again washed in PBS and mounted on glass slides with Fluoromount-G (Southern Biotechnology Associates). Vimentin intermediate fila- 
ments and nuclear morphology were visualized by fluorescence microscopy.

\section{Construction of GFP-tagged vimentin cDNAs}

Full-length and truncated (encoding amino acids 1-85) vimentin cDNAs were PCR amplied from wild-type human vimentin with the following oligonucleotide primers: 5 '-GGCCGAATTTCCCATGTCCACCAGGTCCGTG-3' and 5'-GGCCGGATCCCCTTCAAGGTCATCGTGATG-3' (full-length) or 5'-GGCCGAATTTCCCATGTCCACCAGGTCCGTG-3' and 5'-GGCCGGATCCAAGTCCTGCAG-3' (truncated). The PCR products were then digested with $E c o R I$ and $B a m H I$ and cloned into pEGFP-N1 (Clontech) digested with the same two enzymes. The sequence of the resulting full-length and truncated vimentin constructs fused at their C-termini with GFP was confirmed by automated DNA sequencing.

\section{Transfection experiments}

HeLa and MCF-7 cells were plated on glass cover slips and transfected with $1 \mu \mathrm{g}$ of pEGFP-N1 plasmid containing empty vector, truncated or full-length vimentin using Lipofectamine reagent (Life Technologies) according to the manufacturer's instructions. Twentyfour hours later, cells were fixed in $2.5 \%$ glutaraldehyde and nuclei stained with Hoescht \#33258 as above. The percentage of transfected cells with apoptotic nuclear changes was determined by fluorescence microscopy in triplicate experiments. To determine whether truncated vimentin interferes with assembly of wild-type vimentin into intermediate filaments, MCF-7 cells were co-transfected with pEGFP-N1-truncated vimentin and pEGFP-N1-wild-type vimentin ( $1 \mu \mathrm{g}$ of each plasmid), and transfected cells were analyzed as above. In other co-transfection experiments, HeLa cells were transfected with $0.4 \mu \mathrm{g}$ of pEGFP-N1 plasmid containing truncated vimentin and $1.2 \mu \mathrm{g}$ of various anti-apoptotic cDNAs, and the percentage of transfected cells that were apoptotic was determined as above. The caspase-8 dominant negative cDNA was kindly provided by Drs. I Sanchéz and J Yuan.

\section{Acknowledgements}

We are indebted to Dr. R Talanian for providing the recombinant caspases used in this study, to Drs. I Sanchéz and J Yuan for the caspase- 8 dominant negative cDNA, and to Drs. Harris Perlman and Z Oltvai for their critical reading of the manuscript. This work was supported in part by Mentored Clinical Scientist Development Award K08-CA01752 (to VL Cryns) and R01 AR41836 (to KJ Green) from the NIH, by institutional research grants to Northwestern University from the Howard Hughes Medical Institute and the American Cancer Society (to VL Cryns), and by the Elizabeth Boughton Trust (to VL Cryns).

\section{References}

1. Steller H(1995) Mechanisms and genes of cellular suicide. Science 267: 14451449

2. Cryns VL and Yuan J (1998) Proteases to die for. Genes Dev. 12: 1551 - 1570

3. Thornberry NA and Lazebnik Y (1998) Caspases: enemies within. Science 281 : $1312-1316$

4. Brancolini C, Benedetti M and Schneider C (1995) Microfilament reorganization during apoptosis: the role of Gas2, a possible substrate for ICE-like proteases. EMBO J. 14: $5179-5190$
5. Kothakota S, Azuma T, Reinhard C, Klippel A, Tang J, Chu K, McGarry TJ, Kirschner MW, Koths K, Kwiatkowski DJ and Williams LT (1997) Caspase-3generated fragment of gelsolin: effector of morphological change in apoptosis. Science 278: $294-298$

6. Cryns VL, Bergeron L, Zhu H, LiH and Yuan J (1996) Specific cleavage of $\alpha$-fodrin during Fas- and tumor necrosis factor-induced apoptosis is mediated by an interleukin- $1 \beta$-converting enzyme/Ced-3 protease distinct from the poly(ADPribose) polymerase protease. J. Biol. Chem. 271: 31277-31282

7. Wang K, Posmantur R, Nath R, McGinnis K, Whitton M, Talanian R, Glantz S and Morrow J (1998) Simultaneous degradation of $\alpha$ ll-and $\beta$ II-spectrin by caspase 3 (CPP32) in apoptotic cells. J. Biol. Chem. 273: 22490-22497

8. Lazebnik YA, Takahashi A, Moir RD, Goldman RD, Poirier GG, Kaufmann SH and Earnshaw WC (1995) Studies of the lamin proteinase reveal multiple parallel biochemical pathways during apoptotic execution. Proc. Natl. Acad. Sci. USA 92: 9042-9046

9. Orth K, Chinnaiyan AM, Garg M, Froelich CJ and Dixit VM(1996) The CED-3/ICElike protease Mch2 is activated during apoptosis and cleaves the death substrate lamin A. J. Biol. Chem. 271: 16443-16446

10. Rao L, Perez D and White E (1996) Lamin proteolysis facilitates nuclear events during apoptosis. J. Cell. Biol. 135: 1441-1455

11. Takahashi A, Alnemri ES, Lazebnik YA, Fernandes-Alnemri T, Litwack G, Mori RD, Goldman RD, Poirier GG, Kaufmann SHand Earnshaw WC (1996) Cleavage of lamin A by Mch2 $\alpha$ but not CPP32: multiple interleukin $1 \beta$-converting enzymerelated proteases with distinct substrate recognition properties are active in apoptosis. Proc. Natl. Acad. Sci. USA 93: 8395-8400

12. Caulín C, Salvesen GS and Oshima RG (1997) Caspase cleavage of keratin 18 and reorganization of intermediate filaments during epithelial cell apoptosis. J. Cell. Biol. 138: $1379-1394$

13. Ku N-O, Liao J and Omary MB (1997) Apoptosis generates stable fragments of human type I keratins. J. Biol. Chem. 272: 33197-33203

14. Brancolini C, Lazarevic D and Schneider C (1997) Dismantling cell-cell contacts during apoptosis is coupled to a caspase-dependent proteolytic cleavage of beta-catenin. J. Cell. Biol. 139: 759-771

15. Lustig KD, Stukenberg T, McGarry T, King RW, Cryns VL, Mead P, Zon L, Yuan J and Kirschner MW (1997) Small pool expression screening: a novel strategy for the identification of genes involved in cell cycle control, apoptosis and early development. Methods Enzymol. 283: 83-99

16. Cryns VL, Byun Y, Rana A, Mellor H, Lustig KD, Ghanem L, Parker PJ, Kirschner MW and Yuan J (1997) Specific proteolysis of the kinase protein kinase C-related kinase 2 by caspase-3 during apoptosis: Identification by a novel small pool expression cloning strategy. J. Biol. Chem. 272: 29449-29453

17. Li H, Bergeron L, Cryns V, Pasternack M, Zhu H, Shi L, Greenberg A and Yuan J (1997) Activation of caspase-2 in apoptosis. J. Biol. Chem. 272: 21010-21017

18. Lazebnik YA, Kaufmann SH, Desnoyers S, Poirier GG and Earnshaw WC (1994) Cleavage of poly(ADP-ribose) polymerase by a proteinase with properties like ICE. Nature 371: $346-347$

19. Ferrari S, Battini R, Kaczmarek L, Rittling S, Calabretta B, de Riel J, Philiponis V, Wei J and Baserga R (1986) Coding sequence and growth regulation of the human vimentin gene. Mol. Cell. Biol. 6: 3614-3620

20. Fuchs E and Weber K (1994) Intermediate filaments: structure, dynamics, function, and disease. Annu. Rev. Biochem. 63: 345-382

21. Thornberry N, Rano T, Peterson E, Rasper D, Timkey T, Garcia-Calvo M, Houtzager V, Nordstrom P, Roy S, Vaillancourt J, Chapman K and Nicholson D (1997) A combinatorial approach defines specificities of members of the caspase family and granzyme $B$. Functional relationships established for key mediators of apoptosis. J. Biol. Chem. 272: 17907-17911

22. Talanian RV, Quinlan C, Trautz S, Hackett MC, Mankovich JA, Banach D, Ghayur T, Brady KD and Wong WW (1997) Substrate specificities of caspase family proteases. J. Biol. Chem. 272: 9677-9682

23. Sánchez I, Xu C-J, Juo P, Kakizaka A, Blenis J and Yuan J (1999) Caspase-8 is required for cell death induced by expanded polyglutamine repeats. Neuron 22 : $623-633$

24. Zhou Q, Snipas S, Orth K, Muzio M, Dixit VM and Salvesen GS (1997) Target protease specificity of the viral serpin $\mathrm{CrmA}$. Analysis of five caspases. J. Biol. Chem. 272: $7797-7800$

25. van Engeland $M$, Kuijpers $H$, Ramaekers $F$, Reutelingsperger $C$ and Schutte $B$ (1997) Plasma membrane alterations and cytoskeletal changes in apoptosis. Exp. Cell. Res. 235: $421-430$ 
26. Hashimoto M, Inoue S, Ogawa S, Conrad C, Muramatsu M, Shackelford D and Masliah E (1998) Rapid fragmentation of vimentin in human skin fibroblasts exposed to tamoxifen: A possible involvement of caspase-3. Biochem. Biophys. Res. Commun. 247: 401-406

27. Morishima N (1999) Changes in nuclear morphology during apoptosis correlate with vimentin cleavage by different caspases located either upstream or downstream of Bcl-2 action. Genes to Cells 4: 401-414

28. MacFarlane M, Merrison W, Dinsdale D and Cohen GM (2000) Active caspases and cleaved cytokeratins are sequestered into cytoplasmic inclusions in TRAILinduced apoptosis. J. Cell. Biol. 148: 1239-1254

29. Chou Y-H, Bischoff JR, Beach D and Goldman RD (1990) Intermediate filament reorganization during mitosis is mediated by p34cdc2 phosphorylation of vimentin. Cell 62: 1063-1071
30. Hofmann I and Herrmann $\mathrm{H}$ (1992) Interference in vimentin assembly in vitro by synthetic peptides derived from the vimentin head domain. J. Cell. Sci. 101: $687-700$

31. Yoon M, Moir RD, Prahlad V and Goldman RD (1998) Motile properties of vimentin intermediate filament networks in living cells. J. Cell. Biol. 143: 147157

32. Cardone MH, Salvesen GS, Widmann C, Johnson G and Frisch SM (1997) The regulation of anoikis: MEKK-1 activation requires cleavage by caspases. Cell 90: $315-320$ 